\title{
HOMOGENEOUS INTERPOLATION AND SOME CONTINUED FRACTIONS
}

\author{
IVAN PETRAKIEV
}

Dedicated to Professor Joe Harris on his 60th birthday

\begin{abstract}
We prove: if $d / m<2280 / 721$, there is no curve of degree $d$ passing through $n=10$ general points with multiplicity $m$ in $\mathbf{P}^{2}$. Similar results are given for other special values of $n$. Our bounds can be naturally written as certain palindromic continued fractions.
\end{abstract}

\section{INTRODUCTION}

Denote by $\mathscr{L}(d, n, m)$ the linear system of degree $d$ curves in $\mathbf{P}^{2}$ passing through $n$ general points $\mathscr{P}_{1}, \ldots, \mathscr{P}_{n}$ with multiplicity at least $m$. For $n \geq 9$, Nagata's conjecture $([9])$ predicts that if $\mathscr{L}(d, n, m)$ is nonempty, then $d \geq \sqrt{n} m$. The statement is clear if $n=k^{2}$ is a square, but remains widely open otherwise. Again for $n \geq 9$, a refined conjecture due to Harbourne-Hirschowitz predicts that any linear system $\mathscr{L}(d, n, m)$ is nonspecial, i.e. of dimension $\max \{-1, v\}$, where

$$
v=d(d+3) / 2-n m(m+1) / 2
$$

is the virtual dimension of $\mathscr{L}(d, n, m)$. We refer to [2, [3, [4], 5], 6] for background on the problem and some recent results.

In this paper we prove:

Main Theorem. Let $n$ be a nonsquare positive integer. Write $n=k^{2}+\alpha$ with $k=\lfloor\sqrt{n}\rfloor$. Assume that either:

i) $n=8,10,12$, or

ii) $k \geq 3, \alpha$ is even, $\alpha \mid 2 k$.

If the linear system $\mathscr{L}(d, n, m)$ is nonempty, then $d / m \geq c_{n}^{(2)}$, where

$$
c_{n}^{(2)}=k+\frac{1}{(2 k / \alpha)+\frac{1}{2 k+\frac{1}{(2 k / \alpha)+\frac{1}{k}}} .}
$$

Note that $c_{n}^{(2)}$ is a palindromic continued fraction approximating $\sqrt{n}=[k, \overline{2 k / \alpha, 2 k}]$. The value $c_{8}^{(2)}=\frac{48}{17}$ is well known to be sharp (9]). The next few cases are $c_{10}^{(2)}=\frac{2280}{721}, c_{11}^{(2)}=\frac{660}{199}$ and $c_{12}^{(2)}=\frac{336}{97}$. Similar results were obtained by Biran ([2]) with less terms in the continued fraction, and by Ciliberto et al. ([3]) and Eckl ([6]) also with less terms and only for $n=10$.

Received by the editors December 12, 2012 and, in revised form, November 15, 2013. 2010 Mathematics Subject Classification. Primary 14C20; Secondary 14J26, 14N05. 
As another illustration of our method, we will show that the linear system $\mathscr{L}(1499,10,474)$ with $v=-1$ is nonspecial (Proposition 11.2).

The proof of the Theorem consists of two degenerations. First, we specialize the $n$ general points $\mathscr{P}_{i}$ in $\mathbf{P}^{2}$ to general points $P_{i}$ on a fixed curve $C$ of degree $k$. The problem is naturally reduced to an interpolation problem on a ruled surface $S=\mathbf{P}(\mathscr{E})$ where $\mathscr{E}$ is a semistable rank 2 vector bundle of degree $\alpha$ on $C$. Second, we specialize the $n$ points in $S$ to a curve $\Gamma$ of self-intersection 0 (sometimes this step requires a further degeneration of the underlying surface $S$ ). The methods in this paper extend our previous attempt in [10].

The paper is organized as follows. In Section 2 we introduce a Basic Lemma that will be useful throughout the paper. In Section 3 we perform the first degeneration and obtain a certain weak bound on $d / m$. The construction is formalized in the next two sections. In Section [6 we sketch the proof of the Main Theorem. In each subsequent section we verify the theorem for specific values of $n$. In Section 11 we prove a certain refinement of the Main Theorem. The last section has some auxiliary results on continued fractions.

Notation and conventions. We work over $\mathbb{C}$. For given a subscheme $Y \subset X$ we denote by $\mathscr{N}_{Y / X}=\mathscr{I}_{Y} / \mathscr{I}_{Y}^{2}$ the conormal sheaf of $Y$. For any coherent sheaf $\mathscr{F}$ on $X$, we denote $\mathbf{P}(\mathscr{F})=\operatorname{Proj}\left(\oplus_{\mu \geq 0} \operatorname{Sym}^{\mu} \mathscr{F}\right)$.

\section{BASIC LEMma}

The following elementary lemma is the key ingredient to several arguments in this paper.

Lemma 2.1 (Basic Lemma). Let $C$ be a nonsingular curve embedded in a nonsingular projective variety $X$. Let $D$ be an effective divisor on $X$. Denote $\mu=$ mult $_{C / X}(D)$, the multiplicity of the vanishing of $D$ along $C$. Then, there is a natural injective morphism of sheaves $\mathscr{O}_{C}(-D) \hookrightarrow \mathrm{Sym}^{\mu} \mathscr{N}_{C / X}$, where $\mathscr{N}_{C / X}$ is the conormal bundle of $C$ in $X$.

Proof. Let $\pi: X^{\prime} \rightarrow X$ be the blowup of $C$. Then $\pi^{*}(D)=D^{\prime}+\mu S$, where $S=\mathbf{P}\left(\mathscr{N}_{C / X}\right)$ is the exceptional divisor of $\pi$ and $D^{\prime}$ is the strict transform of $D$. We have $\mathscr{O}_{S}(S)=\mathscr{O}_{S}(-1)$, the tautological line bundle on $S$. So, $\pi_{*}\left(\mathscr{O}_{S}\left(\mu-D^{\prime}\right)\right)=$ $\pi_{*} \pi^{*} \mathscr{O}_{C}(-D)=\mathscr{O}_{C}(-D)$ is naturally a subsheaf of $\pi_{*} \mathscr{O}_{S}(\mu)=\mathrm{Sym}^{\mu} \mathscr{N}_{C / X}$ (also, it is a subbundle iff $\left.D^{\prime}\right|_{S}$ has no vertical components).

We can use the lemma to give a lower bound on $\operatorname{mult}_{C / X}(D)$ based on the "local data" $\mathscr{O}_{C}(-D)$. This takes a particularly simple form if $\mathscr{N}_{C / X}$ is a semistable vector bundle. Recall that, by definition, a vector bundle $\mathscr{E}$ on a curve $C$ is semistable if and only if for any subsheaf $\mathscr{F} \hookrightarrow \mathscr{E}$, we have $\operatorname{slope}(\mathscr{F}) \leq \operatorname{slope}(\mathscr{E})$. Here we denote $\operatorname{slope}(\mathscr{E})=\operatorname{deg}(\mathscr{E}) / \operatorname{rank}(\mathscr{E})$. For background on semistability, see e.g. [1].

Corollary 2.2. In the above setting, suppose that $\mathscr{N}_{C / X}$ is semistable. Then

$$
\operatorname{slope}\left(\mathscr{N}_{C / X}\right) \operatorname{mult}_{C / X}(D) \geq-C \cdot D .
$$

Proof. This follows from the Basic Lemma together with the fact that, for any $\mu \geq 0, \operatorname{Sym}^{\mu} \mathscr{N}_{C / X}$ is semistable of slope $\mu \cdot \operatorname{slope}\left(\mathscr{N}_{C / X}\right)([11$, Theorem 10.2.1). 


\section{FIRST DEGENERATION}

We introduce the first degeneration. As an application we prove Theorem 3.1 below. When $\alpha \mid 2 k$ this result also follows from ([2], Theorem 2.1.A).

Theorem 3.1. Let $n$ be a nonsquare positive integer. Write $n=k^{2}+\alpha$ with $k=\lfloor\sqrt{n}\rfloor$. Assume that either: (i) $\alpha$ is even, or (ii) $k \geq 3$. If the linear system $\mathscr{L}(d, n, m)$ is nonempty, then $d / m \geq c_{n}^{(1)}$, where

$$
c_{n}^{(1)}=k+\frac{1}{(2 k / \alpha)+\frac{1}{k}} .
$$

In particular, the theorem applies for $n=3,6$ and 8 and any $n \geq 10$.

Example 3.2. For $n=3$, we have $c_{3}^{(1)}=\frac{3}{2}$, which is sharp. The linear system $\mathscr{L}(3,3,2)$ has a unique section, namely the union of 3 lines each passing through 2 points ([9], remark on p.772; see also [5], Prop. 2.3).

Example 3.3. If $n=6$, we have $c_{6}^{(1)}=\frac{12}{5}$, which is also sharp. The linear system $\mathscr{L}(12,6,5)$ has a unique section, namely the union of six conics each passing through 5 of the 6 points (Loc. cit.).

Proof of the Theorem. Assume $\mathscr{L}(d, n, m)$ is nonempty. The idea is to specialize the $n$ general points in $\mathbf{P}^{2}$ to a smooth curve $C$ of degree $k$ and then apply the Basic Lemma to estimate the multiplicity of vanishing of a general curve in $\mathscr{L}(d, n, m)$ along $C$.

Step 1 . Let $\Delta$ be the open unit disk over $\mathbb{C}$ and let $X=\mathbf{P}^{2} \times \Delta$. We view $X$ as a relative plane over $\Delta$. For any $t \in \Delta$, denote the fiber $X_{t}=X \times\{t\}$. Fix a smooth curve $C \subset X_{0}$ of degree $k$. We have the following split exact sequence:

$$
\left.0 \rightarrow \mathscr{N}_{X_{0} / X}\right|_{C} \rightarrow \mathscr{N}_{C / X} \rightarrow \mathscr{N}_{C / X_{0}} \rightarrow 0
$$

where $\left.\mathscr{N}_{X_{0} / X}\right|_{C} \cong \mathscr{O}_{C}$ and $\mathscr{N}_{C / X_{0}} \cong \mathscr{O}_{C}(-k H)$. Consider the ruled surface

$$
S^{\prime}=\mathbf{P}\left(\mathscr{N}_{C / X}\right) \text {. }
$$

Let $C^{\prime}$ be the section of $S^{\prime}$ corresponding to the short exact sequence above. Note that $C^{\prime} \sim \mathscr{O}_{S^{\prime}}(1)$ and $\mathscr{O}_{S^{\prime}}\left(C^{\prime}\right) \otimes \mathscr{O}_{C^{\prime}} \cong \mathscr{O}_{C}(-k H)$.

Step 2. Choose any set of $n$ distinct points $P_{i}$ on $C$ (here we do not require the points $P_{i} \in C$ to be general). Next, we construct a set of $n$ relative points $\mathscr{P}_{i} \rightarrow \Delta$ in $X$ specializing to $P_{i}$ in a general way. Denote by $P_{i}^{\prime}$ the images of $\mathscr{P}_{i}$ in $S^{\prime}$. Thus, each $P_{i}^{\prime}$ is a general point on the fiber above $P_{i}$.

Let $\widetilde{X} \rightarrow X$ be the blowup of the relative points $\mathscr{P}_{i}$ and let $E_{i}$ denote the corresponding exceptional divisors. Denote by $\widetilde{C}$ the strict transform of $C$ in $\widetilde{X}$. We have the following short exact sequence:

$$
\left.0 \rightarrow \mathscr{N}_{\widetilde{X}_{0} / \widetilde{X}}\right|_{\widetilde{C}} \rightarrow \mathscr{N}_{\widetilde{C} / \widetilde{X}} \rightarrow \mathscr{N}_{\widetilde{C} / \widetilde{X}_{0}} \rightarrow 0 .
$$

We have $\left.\mathscr{N}_{\widetilde{X}_{0} / \tilde{X}}\right|_{\widetilde{C}} \cong \mathscr{O}_{C}$ and $\mathscr{N}_{\widetilde{C} / \widetilde{X}_{0}} \cong A$, where

$$
A=\mathscr{O}_{C}\left(\sum P_{i}-k H\right)
$$

is a line bundle of degree $\alpha=n-k^{2}$ on $C$. The short exact sequence corresponds to a certain element

$$
\xi \in \operatorname{Ext}^{1}\left(A, \mathscr{O}_{C}\right)
$$


Next, consider the ruled surface

$$
S=\mathbf{P}\left(\mathscr{N}_{\widetilde{C} / \widetilde{X}}\right)
$$

We identify $C$ with the section of $S$ corresponding to the above exact sequence. Note that $C \sim \mathscr{O}_{S}(1)$ and $\mathscr{O}_{S}(C) \otimes \mathscr{O}_{C} \cong A$.

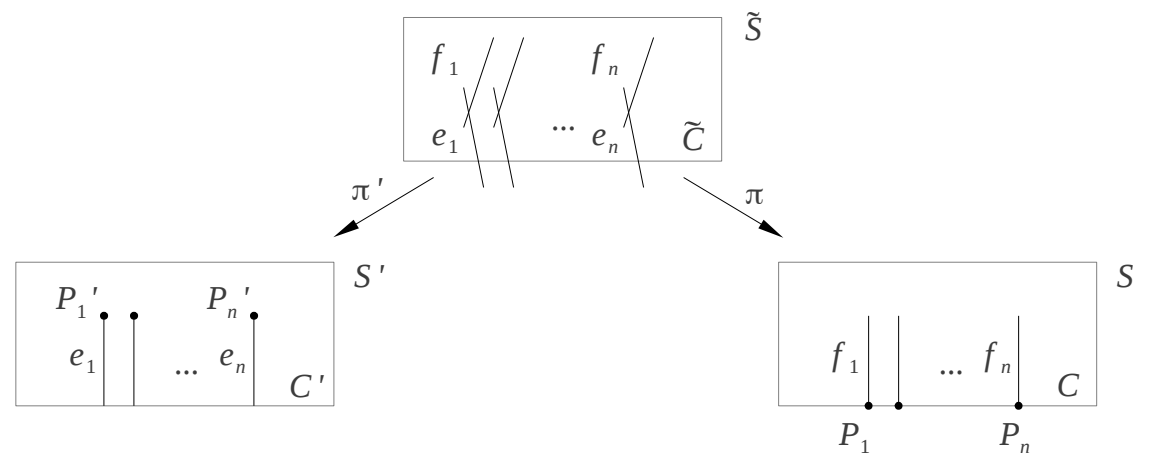

FiguRE 1. Elementary transforms

The conormal bundles $\mathscr{N}_{C / X}$ and $\mathscr{N}_{\widetilde{C} / \widetilde{X}}$ are related by elementary transforms at the points $P_{i}$ :

$$
0 \rightarrow \mathscr{N}_{C / X} \rightarrow \mathscr{N}_{\widetilde{C} / \widetilde{X}} \rightarrow \oplus_{i=1}^{n} \mathbb{C}\left(P_{i}\right) \rightarrow 0 .
$$

Similarly, the ruled surfaces $S^{\prime}$ and $S$ are related by elementary transforms as in Figure 1 (the intermediate surface $\widetilde{S}$ will play a role later in Section 4).

Step 3. We claim:

Lemma 3.4. Let $\xi \in \operatorname{Ext}^{1}\left(A, \mathscr{O}_{C}\right)$ be an arbitrary element. Then $\xi$ can be realized in the above way, for some specialization of points $\mathscr{P}_{i}$ to $P_{i}$.

Proof. The idea is to consider the construction in Step 2 in reversed order. Start with any extension

$$
0 \rightarrow \mathscr{O}_{C} \rightarrow \mathscr{E} \rightarrow A \rightarrow 0
$$

and let $S=\mathbf{P}(\mathscr{E})$. As before, we identify $C$ with the section of $S$ corresponding to the above exact sequence. Next, we construct the vector bundle $\mathscr{E}^{\prime}$ by applying elementary transforms at the points $P_{i}$ on $C$ :

$$
0 \rightarrow \mathscr{E}^{\prime} \rightarrow \mathscr{E} \rightarrow \oplus_{i=1}^{n} \mathbb{C}\left(P_{i}\right) \rightarrow 0 .
$$

Let $S^{\prime}=\mathbf{P}\left(\mathscr{E}^{\prime}\right)$ and let $P_{1}^{\prime}, \ldots, P_{n}^{\prime}$ be as in Figure 1, It follows that $\mathscr{E}^{\prime}$ is realized as an extension

$$
0 \rightarrow \mathscr{O}_{C} \rightarrow \mathscr{E}^{\prime} \rightarrow \mathscr{O}_{C}(-k H) \rightarrow 0
$$

Now, the key observation is that

$$
\operatorname{Ext}^{1}\left(\mathscr{O}_{C}(-k H), \mathscr{O}_{C}\right) \cong H^{1}\left(C, \mathscr{O}_{C}(k H)\right)=0
$$

so the above extension is trivial. This allows us to identify $\mathscr{E}^{\prime} \cong \mathscr{O}_{C} \oplus \mathscr{O}_{C}(-k H)$ with $\mathscr{N}_{C / X}$, and so $S^{\prime}$ with $\mathbf{P}\left(\mathscr{N}_{C / X}\right)$. Finally, we choose the relative points $\mathscr{P}_{i}$ to pass through $P_{i}^{\prime}$ in $S^{\prime}$. This identifies $\mathscr{E}$ with $\mathscr{N}_{\widetilde{C} / \tilde{X}}$, and so $S$ with $\mathbf{P}\left(\mathscr{N}_{\widetilde{C} / \tilde{X}}\right)$. 
Corollary 3.5. If the specialization of $\mathscr{P}_{i}$ to $P_{i}$ is general enough, the conormal bundle $\mathscr{N}_{\widetilde{C} / \widetilde{X}}$ is semistable of slope $\alpha / 2$.

This follows from Lemma 3.4 and the following general fact:

Lemma 3.6. Let $C$ be a curve of genus $g \geq 0$. Let $A$ be a line bundle on $C$ of degree $\alpha \geq 0$. Assume that either: (i) $\alpha$ is even, or (ii) $g \geq 1$. Then, a general element $\xi \in \operatorname{Ext}^{1}\left(A, \mathscr{O}_{C}\right)$ corresponds to a semistable rank 2 vector bundle $\mathscr{E}$ on $C$.

Proof. The set of elements $\xi \in \operatorname{Ext}^{1}\left(A, \mathscr{O}_{C}\right)$ that correspond to semistable vector bundles $\mathscr{E}$ is open. So, it suffices to show that the set is nonempty. Now, if $g=0$ and $\alpha=2 \alpha_{0}$ is even, then $\mathscr{O}_{\mathbf{P}^{1}}\left(\alpha_{0}\right) \oplus \mathscr{O}_{\mathbf{P}^{1}}\left(\alpha_{0}\right)$ is semistable. If $g \geq 1$, one can prove the statement by induction on $\alpha$. We leave this as an exercise.

Step 4 . We complete the proof of the theorem. Since $\mathscr{L}(d, n, m)$ is nonempty by assumption, there is a flat family of curves $\tilde{\mathscr{C}} \rightarrow \Delta$, where $\tilde{\mathscr{C}}$ is a nontrivial section of $\left|\mathscr{O}_{\widetilde{X}}\left(d H-\sum m E_{i}\right)\right|$. We are interested in estimating $\mu=\operatorname{mult}_{\widetilde{C} / \widetilde{X}}(\widetilde{\mathscr{C}})$, which of course is the same as $\mu=\operatorname{mult}_{C / X}(\mathscr{C})$ where $\mathscr{C}$ is the image of $\tilde{\mathscr{C}}$ in $X$. Obviously,

$$
\mu \leq \frac{d}{k}
$$

By Corollaries 3.5 and 2.2, we have:

$$
\underbrace{\operatorname{slope}\left(\mathscr{N}_{\widetilde{C} / \widetilde{X}}\right)}_{\alpha / 2} \cdot \mu \geq \underbrace{-\widetilde{C} \cdot \tilde{\mathscr{C}}}_{n m-k d} .
$$

Combining $(\sharp)$ and $(b)$, we get:

$$
\frac{\alpha}{2} \frac{d}{k} \geq n m-k d
$$

One can easily check that this is equivalent to the inequality in the theorem. See also Lemma 13.1(a).

\section{Reduction of interpolation PROBlems to RULED SURFACES}

We formalize some results from the previous section. Our result here is Theorem 4.5 which will be used throughout the rest of the paper.

Notation 4.1. A marked surface $\left(S ; P_{1}, \ldots, P_{n}\right)$ is simply a surface $S$ together with $n$ distinct points $P_{1}, \ldots, P_{n}$ on $S$.

Notation 4.2. Let $S=\left(\mathbf{P}(\mathscr{E}) ; P_{1}, \ldots, P_{n}\right)$ be a marked ruled surface over $C$. For any integers $(\mu, b, \widehat{m})$ and a line bundle $\mathfrak{b}$ of degree $b$ on $C$, we denote the line bundle

$$
\mathscr{L}_{S}(\mu, \mathfrak{b}, \widehat{m})=\mathscr{O}_{\widetilde{S}}\left(\mu-\mathfrak{b} f-\sum \widehat{m} e_{i}\right)
$$

on the blowup $\pi: \widetilde{S} \rightarrow S$ at the points $P_{1}, \ldots, P_{n}$, with exceptional divisors $e_{1}, \ldots, e_{n}$. Here we denote $\mathscr{O}_{\widetilde{S}}(\mu)=\pi^{*} \mathscr{O}_{S}(\mu)$.

Lemma 4.3. We have

$$
\chi\left(\mathscr{L}_{S}(\mu, \mathfrak{b}, \widehat{m})\right)=(\mu+1)\left(\frac{\alpha}{2} \mu-b-g+1\right)-n\left(\begin{array}{c}
\widehat{m}+1 \\
2
\end{array}\right)
$$

where $\alpha=\operatorname{deg}(\mathscr{E})$ and $g$ is the genus of $C$. 
Proof. Denote by $C_{1}$ the class $c_{1}\left(\mathscr{O}_{S}(1)\right)$. It follows from ([8], Lemma V.2.10), that

$$
K_{S} \equiv-2 C_{1}+(2 g-2+\alpha) f .
$$

By the Riemann-Roch formula,

$$
\chi\left(\mathscr{O}_{S}\left(\mu C_{1}-\mathfrak{b} f\right)\right)=\frac{1}{2}\left(\mu C_{1}-\mathfrak{b} f\right) \cdot\left(\mu C_{1}-\mathfrak{b} f-K_{S}\right)+1+p_{a}(S) .
$$

We have $p_{a}(S)=-g\left([8]\right.$, Cor. V.2.5). The lemma now follows from $C_{1}^{2}=\alpha, C_{1} \cdot f=$ 1 and $f^{2}=0$.

Notation 4.4. Let $C$ be a smooth curve, $A$ a line bundle on $C$ and let $\xi \in \operatorname{Ext}^{1}\left(A, \mathscr{O}_{C}\right)$ correspond to an extension

$$
0 \rightarrow \mathscr{O}_{C} \rightarrow \mathscr{E} \rightarrow A \rightarrow 0
$$

We denote by

$$
S(C, A, \xi)
$$

the ruled surface $S=\mathbf{P}(\mathscr{E})$ and we identify $C$ with the section determined by the short exact sequence. Note that $C \sim \mathscr{O}_{S}(1)$ and $\mathscr{O}_{S}(C) \otimes \mathscr{O}_{C} \cong A$.

The following theorem allows us to reduce interpolation problems on $\mathbf{P}^{2}$ to certain interpolation problems on ruled surfaces.

Theorem 4.5. Let $n=k^{2}+\alpha$ where $k$ and $\alpha$ are positive integers. Consider the linear system $\mathscr{L}(d, n, m)$ for some positive integers $d$ and $m$. Fix a smooth curve $C$ of degree $k$ in $\mathbf{P}^{2}$. Let $S=S\left(C, A, \xi ;\left\{P_{i}\right\}\right)$ be a marked ruled surface where:

- $A$ is any line bundle of degree $\alpha$ on $C$;

- $\xi \in \operatorname{Ext}^{1}\left(A, \mathscr{O}_{C}\right)$ is any element;

- $P_{1}, \ldots, P_{n}$ are distinct points on $C \subset S$ such that $\sum P_{i} \sim A+k H$.

Then, for any $\mu$, we have

$$
h^{0}\left(\mathscr{L}_{\mathbf{P}^{2}}(d, n, m)\right) \leq h^{0}\left(\mathscr{L}_{S}(\mu, \mathfrak{b}, \widehat{m})\right)+h^{0}\left(\mathscr{O}_{\mathbf{P}^{2}}(\epsilon H)\right)-h^{0}\left(\mathscr{O}_{C}(\epsilon H)\right)
$$

where:

- $\mathfrak{b}=\mathscr{O}_{C}\left(\sum m P_{i}-d H\right)$ of degree $b=n m-k d$;

- $\widehat{m}=\mu-m$;

- $\epsilon=d-k \mu$.

The following lemma justifies our definition of $\mathscr{L}_{S}(\mu, \mathfrak{b}, \widehat{m})$ :

Lemma 4.6. In the setting of the theorem, we have $\mathscr{L}_{S}(\mu, \mathfrak{b}, \widehat{m}) \otimes \mathscr{O}_{\widetilde{C}} \cong \mathscr{O}_{C}(\epsilon H)$, where $\widetilde{C}$ is the strict transform of $C$ in $\widetilde{S}$.

Proof. This is an easy computation. We have:

$$
\mathscr{O}_{S}(\mu C-\mathfrak{b} f) \otimes \mathscr{O}_{C} \cong \mathscr{O}_{C}(\mu A-\mathfrak{b}) \cong \mathscr{O}_{C}\left(\sum \widehat{m} P_{i}+\epsilon H\right)
$$

The lemma follows.

Proof of Theorem 4.5. We will use the same construction as in the proof of Theorem 3.1 
Step 1. Consider the threefold $X=\mathbf{P}^{2} \times \Delta$. We identify $C$ with a curve on $X_{0}$. Given $\xi \in \operatorname{Ext}^{1}\left(A, \mathscr{O}_{C}\right)$, we specialize the $n$ relative points $\mathscr{P}_{i}$ to $P_{i} \in C$ as in Lemma 3.4, As before, let $\widetilde{X} \rightarrow X$ be the blowup of the $\mathscr{P}_{i}$, and let $\widetilde{C}$ be the strict transform of $C$ in $\widetilde{X}$. We have the short exact sequence

$$
\left.0 \rightarrow \mathscr{N}_{\widetilde{X}_{0} / \tilde{X}}\right|_{\widetilde{C}} \rightarrow \mathscr{N}_{\widetilde{C} / \widetilde{X}} \rightarrow \mathscr{N}_{\widetilde{C} / \widetilde{X}_{0}} \rightarrow 0
$$

where $\left.\mathscr{N}_{\widetilde{X}_{0} / \widetilde{X}}\right|_{\widetilde{C}} \cong \mathscr{O}_{C}$ and $\mathscr{N}_{\widetilde{C} / \widetilde{X}_{0}} \cong A$. By construction, the above extension corresponds to $\xi$. In particular, $S \cong \mathbf{P}\left(\mathscr{N}_{\widetilde{C} / \widetilde{X}}\right)$, where $S=S(C, A, \xi)$ is the ruled surface we started with.

Step 2. Consider the threefold $Y$ obtained from $X=\mathbf{P}^{2} \times \Delta$ by first blowing up $C$ (with exceptional divisor $S^{\prime}=\mathbf{P}\left(\mathscr{N}_{C / X}\right)$ ), followed by blowing up the strict transforms of the relative points $\mathscr{P}_{1}, \ldots, \mathscr{P}_{n}$ (with exceptional divisors $E_{1}, \ldots, E_{n}$ ). We view $Y \rightarrow \Delta$ as a flat family with general fiber $Y_{t} \cong \widetilde{X}_{t}$. The special fiber $Y_{0}$ is the union of two surfaces $\widetilde{S} \cup X_{0}$ meeting transversely along $\widetilde{C} \cong C \mathbb{1}$

This construction is related to the construction in Step 1 as follows. Let $\widetilde{X}^{\prime}$ be the threefold obtained from $\widetilde{X}$ by blowing up $\widetilde{C}$ (with exceptional divisor $S=$ $\left.\mathbf{P}\left(\mathscr{N}_{\widetilde{C} / \tilde{X}}\right)\right)$. Then, $Y$ can be obtained from $\widetilde{X}^{\prime}$ by applying (-1)-transfers to the exceptional curves $e_{1}, \ldots, e_{n}$ as in Figure 2 (see also [4, Section 4.1). The induced map $\pi: \widetilde{S} \rightarrow S$ coincides with the corresponding map in Figure 1.
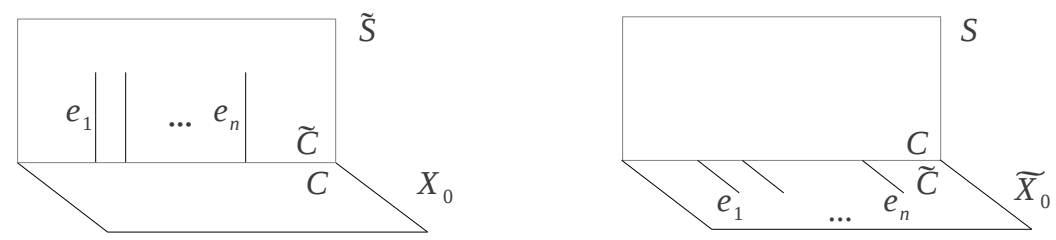

Figure 2. (-1) transfers between $Y$ and $\widetilde{X}^{\prime}$

Step 3. For a given $\mu$, consider the following line bundle on $Y$ :

$$
\mathscr{L}_{Y} \cong \mathscr{O}_{Y}\left(d H-\sum m E_{i}-\mu \widetilde{S}\right) \text {. }
$$

We view $\mathscr{L}_{Y}$ as a flat family of line bundles with general fiber $\mathscr{L}_{Y_{t}} \cong \mathscr{O}_{\widetilde{X}_{t}}(d H-$ $\left.\sum m E_{i}\right)$. The special fiber $\mathscr{L}_{Y_{0}}$ is described by the following short exact sequence:

$$
0 \rightarrow \mathscr{L}_{Y_{0}} \rightarrow \mathscr{L}_{\widetilde{S}} \oplus \mathscr{L}_{X_{0}} \rightarrow \mathscr{L}_{C} \rightarrow 0
$$

where

$$
\mathscr{L}_{\widetilde{S}} \cong \mathscr{L}_{Y} \otimes \mathscr{O}_{\widetilde{S}} ; \quad \mathscr{L}_{X_{0}} \cong \mathscr{O}_{\mathbf{P}^{2}}(\epsilon H) ; \quad \mathscr{L}_{C} \cong \mathscr{O}_{C}(\epsilon H)
$$

Lemma 4.7. We have:

$$
\mathscr{L}_{\widetilde{S}} \cong \mathscr{L}_{S}(\mu, \mathfrak{b}, \widehat{m})
$$

\footnotetext{
${ }^{1}$ We denote by $\widetilde{C}$, resp. $C$, the same curve in $Y$ viewed as a divisor in $\widetilde{S}$, resp. $X_{0}$.
} 
Proof. The line bundle $\mathscr{L}_{\widetilde{S}}$ has the following properties:

$$
\mathscr{L}_{\widetilde{S}} \otimes \mathscr{O}_{\widetilde{C}} \cong \mathscr{O}_{C}(\epsilon H) ; \quad \mathscr{L}_{\widetilde{S}} \cdot f=\mu ; \quad \mathscr{L}_{\widetilde{S}} \cdot e_{i}=\widehat{m} .
$$

From Lemma 4.6, $\mathscr{L}_{S}(\mu, \mathfrak{b}, \widehat{m})$ has the same properties. It follows that $\mathscr{L}_{\widetilde{S}} \cong$ $\mathscr{L}_{S}(\mu, \mathfrak{b}, \widehat{m})$.

To complete the proof of the theorem, take cohomology in $(*)$ :

$$
0 \rightarrow H^{0}\left(\mathscr{L}_{Y_{0}}\right) \rightarrow H^{0}\left(\mathscr{L}_{\widetilde{S}}\right) \oplus H^{0}\left(\mathscr{O}_{\mathbf{P}^{2}}(\epsilon H)\right) \rightarrow H^{0}\left(\mathscr{O}_{C}(\epsilon H)\right) \stackrel{\delta}{\rightarrow} H^{1}\left(\mathscr{L}_{Y_{0}}\right) .
$$

The restriction $H^{0}\left(\mathscr{O}_{\mathbf{P}^{2}}(\epsilon H)\right) \rightarrow H^{0}\left(\mathscr{O}_{C}(\epsilon H)\right)$ is surjective. Hence, the coboundary map $\delta=0$. The theorem now follows from the semicontinuity principle applied to $h^{0}\left(\mathscr{L}_{Y_{t}}\right)$.

Corollary 4.8. Assume the above setting.

(a) For any $\mu$, we have:

$$
\chi\left(\mathscr{L}_{\mathbf{P}^{2}}(d, n, m)\right)=\chi\left(\mathscr{L}_{S}(\mu, \mathfrak{b}, \widehat{m})\right)+\chi\left(\mathscr{O}_{\mathbf{P}^{2}}(\epsilon H)\right)-\chi\left(\mathscr{O}_{C}(\epsilon H)\right) .
$$

(b) For $\mu=\lfloor d / k\rfloor$, we have:

$$
\chi\left(\mathscr{L}_{\mathbf{P}^{2}}(d, n, m)\right)=\chi\left(\mathscr{L}_{S}(\mu, \mathfrak{b}, \widehat{m})\right)+h^{1}\left(\mathscr{O}_{C}(\epsilon H)\right) .
$$

Proof. (a) This follows from the short exact sequence $(*)$ and the fact that $\chi\left(\mathscr{L}_{Y_{t}}\right)$ is a constant function of $t$.

(b) This follows from (a).

Corollary 4.9. Suppose the linear system $\mathscr{L}(d, n, m)$ is nonempty. Then, with $\mu=\lfloor d / k\rfloor,\left|\mathscr{L}_{S}(\mu, \mathfrak{b}, \widehat{m})\right|$ is nonempty.

Proof. Consider the long exact cohomology sequence associated to $(*)$. If $\mu=$ $\lfloor d / k\rfloor$, the restriction $H^{0}\left(\mathscr{O}_{\mathbf{P}^{2}}(\epsilon H)\right) \rightarrow H^{0}\left(\mathscr{O}_{C}(\epsilon H)\right)$ is an isomorphism. The claim follows.

\section{FAMilies OF RULED SURFACES}

We can use the degeneration technique from the previous section to reduce an interpolation problem on $\mathbf{P}^{2}$ to an interpolation problem on a certain ruled surface $S$. We would like to perform further degenerations to study the later problem. Our first goal is to define an object $\mathfrak{S}(Z, \mathscr{A}, \xi) \rightarrow \Delta$ which is a relative analogue of $S(C, A, \xi)$. We conclude with a technical result (Proposition 5.4) which will be used in Sections 9 and 12. As usual, $\Delta$ denotes the open unit disk over $\mathbb{C}$.

Notation 5.1. Let $\mathscr{A}$ be a torsion-free sheaf of rank 1 on $C \times \Delta$. Assume that $\mathscr{A}=\mathscr{A}^{\prime} \otimes \mathscr{I}_{W}$ where $\mathscr{A}^{\prime}$ is invertible and $\mathscr{I}_{W}$ is the ideal sheaf of an l.c.i. zerodimensional subscheme $W \subset C \times \Delta$ (possibly $W=\emptyset)$. Let $\xi \in \operatorname{Ext}^{1}\left(\mathscr{A}, \mathscr{O}_{C \times \Delta}\right)$ be an element corresponding to an extension

$$
0 \rightarrow \mathscr{O}_{C \times \Delta} \rightarrow \mathscr{E} \rightarrow \mathscr{A} \rightarrow 0
$$

such that $\mathscr{E}$ is locally free. We denote by

$$
\mathfrak{S}(Z, \mathscr{A}, \xi) \rightarrow \Delta
$$

the relative ruled surface $\mathfrak{S}=\mathbf{P}(\mathscr{E}) \rightarrow \Delta$ together with the subscheme $Z=\mathbf{P}(\mathscr{A})$ defined by the above exact sequence. In particular, $Z$ is a divisor of $\mathfrak{S}$ with $Z \sim$ $\mathscr{O}_{\mathfrak{S}}(1)$. 
We will assume that $W$ is supported on $C \times\{0\}$. In applications, $W$ will be reduced; however, everything we say in this section holds in the more general setting.

Lemma 5.2. In the above setting, the projection $p: Z \rightarrow C \times \Delta$ is just the blowup of $W \subset C \times \Delta$. Denote by $F$ the exceptional divisor of $p$. Then $p^{*} \mathscr{A} \cong$ $p^{*} \mathscr{A}^{\prime} \otimes \mathscr{O}_{Z}(-F) \cong \mathscr{O}_{\mathfrak{S}}(Z) \otimes \mathscr{O}_{Z}$

Proof. Since $\mathscr{A}^{\prime}$ is invertible, $Z=\mathbf{P}(\mathscr{A})=\mathbf{P}\left(\mathscr{I}_{W}\right)$ which is exactly the definition of a blowup. The rest is clear.

Consider the following question: given $\mathscr{A}$ as above, which elements $\xi \in$ $\operatorname{Ext}^{1}\left(\mathscr{A}, \mathscr{O}_{C \times \Delta}\right)$ correspond to a locally free extension $\mathscr{E}$ ? Clearly, if $W=\emptyset$, then $\mathscr{A}$ is locally free and so any $\xi$ will do. In the general case, we have:

Proposition 5.3. Let $\mathscr{A}=\mathscr{A}^{\prime} \otimes \mathscr{I}_{W}$ be as above. Then, there is a natural exact sequence

$$
0 \rightarrow H^{1}\left(C \times \Delta,\left(\mathscr{A}^{\prime}\right)^{-1}\right) \rightarrow \operatorname{Ext}^{1}\left(\mathscr{A}, \mathscr{O}_{C \times \Delta}\right) \stackrel{\delta}{\rightarrow} H^{0}\left(\mathscr{E} x t^{1}\left(\mathscr{A}, \mathscr{O}_{C \times \Delta}\right)\right) \rightarrow 0 .
$$

Moreover, we have:

(a) $\mathscr{E} x t^{1}\left(\mathscr{A}, \mathscr{O}_{C \times \Delta}\right) \cong \mathscr{E} x t^{2}\left(\mathscr{O}_{W}, \mathscr{O}_{C \times \Delta}\right) \cong \mathscr{O}_{W}$

(b) The extension $\mathscr{E}$ corresponding to $\xi \in \operatorname{Ext}^{1}\left(\mathscr{A}, \mathscr{O}_{C \times \Delta}\right)$ is locally free if and only if $\delta(\xi)$ generates the sheaf $\mathscr{E} x t^{1}\left(\mathscr{A}, \mathscr{O}_{C \times \Delta}\right)$.

Proof. See [7, Chapter 2, pp. 36-37. The exact sequence follows from the localto-global spectral sequence

$$
E_{2}^{i, j}=H^{i}\left(\mathscr{E} x t^{j}\left(\mathscr{A}, \mathscr{O}_{C \times \Delta}\right)\right) \Longrightarrow \operatorname{Ext}^{i+j}\left(\mathscr{A}, \mathscr{O}_{C \times \Delta}\right) .
$$

Note that $E_{2}^{i, 0}=H^{i}\left(\mathscr{A}^{-1}\right)=H^{i}\left(\left(\mathscr{A}^{\prime}\right)^{-1}\right)=H^{0}\left(\Delta, R^{i} \pi_{*}\left(\mathscr{A}^{\prime}\right)^{-1}\right)$ where $\pi: C \times$ $\Delta \rightarrow \Delta$ is the projection. In particular, $E_{2}^{i, 0}=0$ for $i \geq 2$.

(a) Ibid., Lemma 7 (note that the isomorphisms are not canonical).

(b) Ibid., Theorem 8 .

Consider a relative ruled surface $\mathfrak{S}(Z, \mathscr{A}, \xi) \rightarrow \Delta$. For any $t \in \Delta, \mathfrak{S}_{t}=\mathbf{P}\left(\mathscr{E}_{t}\right)$ is a ruled surface over $C \times\{t\}$ where $\mathscr{E}_{t}$ arises as an extension

$$
0 \rightarrow \mathscr{O}_{C \times\{t\}} \rightarrow \mathscr{E}_{t} \rightarrow \mathscr{A}_{t} \rightarrow 0 .
$$

We have $Z_{t} \sim \mathscr{O}_{\mathfrak{S}_{t}}(1)$. For a general $t$, the subscheme $Z_{t}$ is a section of $\mathfrak{S}_{t}$. On the special fiber, we have $Z_{0}=C_{0} \cup F_{0}$, where $C_{0}$ is a section of $\mathfrak{S}_{0}$ and $F_{0}=F \times\{0\}$ is the vertical component of $Z_{0}$. If $F_{0} \neq \emptyset$, we will say that $Z_{0}$ is a degenerate section of $\mathfrak{S}_{0}$.

Next, we will show that any degenerate section can be smoothed, in the following sense.

Proposition 5.4. Let $\mathscr{A}=\mathscr{A}^{\prime} \otimes \mathscr{I}_{W}$ be as above, with $W$ supported on $C \times\{0\}$. Let

$$
0 \rightarrow \mathscr{O}_{C \times\{0\}} \rightarrow \mathscr{E}_{0} \rightarrow \mathscr{A}_{0} \rightarrow 0
$$

be any extension, with $\mathscr{E}_{0}$ locally free. Then, the exact sequence can be extended to

$$
0 \rightarrow \mathscr{O}_{C \times \Delta} \rightarrow \mathscr{E} \rightarrow \mathscr{A} \rightarrow 0
$$

with $\mathscr{E}$ locally free on $C \times \Delta$. 
Proof. Consider the commutative diagram with exact rows:

$$
\begin{aligned}
& 0 \rightarrow H^{1}\left(C \times \Delta,\left(\mathscr{A}^{\prime}\right)^{-1}\right) \rightarrow \operatorname{Ext}^{1}\left(\mathscr{A}, \mathscr{O}_{C \times \Delta}\right) \rightarrow H^{0}\left(\mathscr{E} x t^{1}\left(\mathscr{A}, \mathscr{O}_{C \times \Delta}\right)\right) \rightarrow 0 \\
& 0 \rightarrow H^{1}\left(C \times\{0\},\left(\mathscr{A}_{0}\right)^{-1}\right) \rightarrow \operatorname{Ext}^{1}\left(\mathscr{A}_{0}, \mathscr{O}_{C \times\{0\}}\right) \rightarrow H^{0}\left(\mathscr{E} x t^{1}\left(\mathscr{A}_{0}, \mathscr{O}_{C \times\{0\}}\right)\right) \rightarrow 0 .
\end{aligned}
$$

Note that $\mathscr{A}_{0} \cong \mathscr{A}_{0}^{\prime}\left(-W_{0}\right) \oplus \mathscr{O}_{W_{0}}$ where $W_{0}=W \times\{0\}$. Therefore, $\left(\mathscr{A}_{0}\right)^{-1} \cong$ $\left(\mathscr{A}_{0}^{\prime}\left(-W_{0}\right)\right)^{-1}$. It follows that the bottom row of the diagram splits. Now, the map on the left factors through

$$
\underbrace{H^{1}\left(C \times \Delta,\left(\mathscr{A}^{\prime}\right)^{-1}\right)}_{H^{0}\left(\Delta, R^{1} \pi_{*}\left(\mathscr{A}^{\prime}\right)^{-1}\right)} \rightarrow \underbrace{H^{1}\left(C \times\{0\},\left(\mathscr{A}_{0}^{\prime}\right)^{-1}\right)}_{\left(R^{1} \pi_{*}\left(\mathscr{A}^{\prime}\right)^{-1}\right)_{0}} \rightarrow H^{1}\left(C \times\{0\},\left(\mathscr{A}_{0}\right)^{-1}\right)
$$

which is surjective. The map on the right is just the restriction

$$
H^{0}\left(\mathscr{O}_{W}\right) \rightarrow H^{0}\left(\mathscr{O}_{W_{0}}\right)
$$

which is also surjective. By the Short Five Lemma, the map in the middle is surjective as well. Hence, any given extension $\xi_{0} \in \operatorname{Ext}^{1}\left(\mathscr{A}_{0}, \mathscr{O}_{C \times\{0\}}\right)$ can be lifted to $\xi \in \operatorname{Ext}^{1}\left(\mathscr{A}, \mathscr{O}_{C \times \Delta}\right)$. The resulting $\mathscr{E}$ is locally free by Proposition 5.3 (b).

\section{Main Result - OVERVieW}

The following theorem was announced in the introduction. The proof will occupy the rest of the paper. We will consider a certain refinement in Section 11.

Main Theorem. Let $n$ be a nonsquare positive integer. Write $n=k^{2}+\alpha$ with $k=\lfloor\sqrt{n}\rfloor$. Assume that either:

i) $n=8,10,12$, or

ii) $k \geq 3, \alpha$ is even, $\alpha \mid 2 k$.

If the linear system $\mathscr{L}(d, n, m)$ is nonempty, then $d / m \geq c_{n}^{(2)}$.

The proof of the theorem consists of the four steps outlined below.

6.1. Setup. We assume $\mathscr{L}(d, n, m)$ is nonempty. Fix a smooth curve $C$ of degree $k$ in $\mathbf{P}^{2}$. By Corollary 4.9, the linear system $\left|\mathscr{L}_{S}(\mu, \mathfrak{b}, \widehat{m})\right|$ is nonempty, with $\mu=$ $\lfloor d / k\rfloor$, for any marked ruled surface $S\left(C, A, \xi ;\left\{P_{i}\right\}\right)$ as in Theorem 4.5 .

6.2. Degeneration. We will construct a relative marked ruled surface $\mathfrak{S}\left(Z, \mathscr{A}, \xi ;\left\{\mathscr{P}_{i}\right\}\right)$ over $\Delta$ such that the general fiber $\mathfrak{S}_{t}$ satisfies the assumptions of Theorem 4.5 i.e.:

- $\mathscr{A}$ is a line bundle on $C \times \Delta$ of relative degree $\alpha=\operatorname{deg} \mathscr{A}_{t}$.

- $\mathscr{P}_{1}, \ldots, \mathscr{P}_{n}$ lie on $Z$. We denote by $\overline{\mathscr{P}}_{i}$ the projection of $\mathscr{P}_{i}$ under $Z \rightarrow$ $C \times \Delta$.

- For $t \in \Delta$ general, we have $\sum \mathscr{P}_{i, t} \sim \mathscr{A}_{t}+k H$ on $Z_{t} \cong C$.

We now describe the special fiber $\mathfrak{S}_{0}$. We assume that $\mathfrak{S}_{0}$ is semistable. Next, we assume that there is a smooth (possibly disconnected) curve $\Gamma$ on $\mathfrak{S}_{0}$ with the following two properties:

- $\Gamma$ meets $Z_{0}$ transversely at $n$ distinct points $P_{i}=\mathscr{P}_{i, 0}$;

- $\Gamma^{2}=0$. 
This determines uniquely the numerical class of $\Gamma$ :

$$
\Gamma \equiv \frac{2 n}{\alpha}\left(Z_{0}-\frac{\alpha}{2} f\right)
$$

where $Z_{0} \sim \mathscr{O}_{\mathfrak{S}_{0}}(1)$.

Let $\Gamma=\sum_{i=1}^{s} \Gamma_{i}$ where each $\Gamma_{i}$ is a smooth irreducible curve, with $\Gamma_{i} \cdot \Gamma_{j}=0$ for $i \neq j$. Since $\Gamma^{2}=0$ and $\mathfrak{S}$ is semistable, $\Gamma$ lies on the boundary of the effective cone of $\mathfrak{S}_{0}$. Therefore, $\Gamma_{i} \equiv \lambda_{i} \Gamma$ for some $\lambda_{i} \in \mathbb{Q}$ with $\sum \lambda_{i}=1$ (in fact, in applications we will always have $\lambda_{1}=\cdots=\lambda_{s}=1 / s$ ).

6.3. Semistability. Denote by $\pi: \widetilde{\mathfrak{S}} \rightarrow \mathfrak{S}$ the blowup of $\mathscr{P}_{1}, \ldots, \mathscr{P}_{n}$ and let $E_{1}, \ldots, E_{n}$ be the corresponding exceptional divisors. Denote by $\widetilde{\Gamma}$ the strict transform of $\Gamma$. We make the following hypothesis:

- for each $i$, the conormal bundle $\mathscr{N}_{\widetilde{\Gamma}_{i} / \widetilde{S}}$ is semistable of slope $\frac{1}{2} \lambda_{i} n$.

6.4. Invariants. Assuming the construction above can be realized, we complete the proof of the theorem. Consider the line bundle

$$
\mathscr{L}_{\mathfrak{S}}(\mu, \mathscr{B}, \widehat{m})=\mathscr{O}_{\widetilde{\mathfrak{S}}}\left(\mu-\mathscr{B} f-\sum \widehat{m} E_{i}\right)
$$

on the blowup $\pi: \widetilde{\mathfrak{S}} \rightarrow \mathfrak{S}$, where

- $\mu=\lfloor d / k\rfloor$;

- $\mathscr{B}=\mathscr{O}_{C \times \Delta}\left(\sum m \overline{\mathscr{P}}_{i}-d H\right)$ of relative degree $b=\operatorname{deg} \mathscr{B}_{t}=n m-k d$.

- $\widehat{m}=\mu-m$.

By construction, there is a flat family of curves $\widetilde{\mathscr{C}} \rightarrow \Delta$ in $\widetilde{\mathfrak{S}}$, where $\widetilde{\mathscr{C}}$ is a section of $\left|\mathscr{L}_{\mathfrak{S}}(\mu, \mathscr{B}, \widehat{m})\right|$. Denote by $\mathscr{C}$ the projection of $\tilde{\mathscr{C}}$ in $\mathfrak{S}$. The following is a key computation:

Lemma 6.1. Define $\gamma=-\frac{1}{n} \widetilde{\Gamma} \cdot \widetilde{\mathscr{C}}$. Then

$$
\gamma=\frac{n+k^{2}}{\alpha} m-\frac{2 k}{\alpha} d
$$

Proof. Using that $\Gamma \cdot Z_{0}=n, \Gamma \cdot f=\frac{2 n}{\alpha}$ and $\widetilde{\mathscr{C}}_{0} \sim \mu Z_{0}-\mathfrak{b} f-\sum \widehat{m} E_{i, 0}$, we find:

$$
\begin{aligned}
\gamma & =-\frac{1}{n} \widetilde{\Gamma} \cdot \widetilde{\mathscr{C}} \\
& =-\frac{1}{n}(\Gamma \cdot \mathscr{C}-n \widehat{m}) \\
& =-\left(\mu-\frac{2}{\alpha} b\right)+\widehat{m} \\
& =-m+\frac{2}{\alpha} b .
\end{aligned}
$$

Finally, substitute $b=n m-k d$ and $n=k^{2}+\alpha$.

To complete the proof of the theorem, we will estimate $\mu_{i}=\operatorname{mult}_{\Gamma_{i} / \mathfrak{S}}(\mathscr{C})$ in two ways. First, there is an obvious upper bound which comes from the numerical class of $\Gamma$ :

$$
\sum \lambda_{i} \mu_{i} \leq \frac{\mu}{2 n / \alpha}
$$

Since $\mu \leq d / k$, this becomes:

$$
\sum \lambda_{i} \mu_{i} \leq \frac{d}{2 k n / \alpha}
$$


By the Basic Lemma and the semistability hypothesis, we have:

$$
\underbrace{\operatorname{slope}\left(\mathscr{N}_{\widetilde{\Gamma}_{i} / \widetilde{\mathfrak{S}}}\right)}_{\frac{1}{2} \lambda_{i} n} \cdot \mu_{i} \geq \underbrace{-\widetilde{\Gamma}_{i} \cdot \tilde{\mathscr{C}}}_{\lambda_{i} n \gamma}
$$

i.e.

$$
\frac{1}{2} \mu_{i} \geq \gamma
$$

From $(\sharp)$ and $(b)$, and using $\sum \lambda_{i}=1$, we get:

$$
\frac{1}{2} \frac{d}{2 k n / \alpha} \geq \gamma=\frac{n+k^{2}}{\alpha} m-\frac{2 k}{\alpha} d .
$$

By Lemma 13.1(b), this is equivalent to the inequality in the theorem.

\section{Eight POINTS}

We verify the Main Theorem in the case $n=8$. The value $c_{8}^{(2)}=\frac{48}{17}$ is well known to be sharp (see example below). We include this case for illustration purposes.

7.1. Setup. Since $k=2$, we take $C$ to be a smooth conic in $\mathbf{P}^{2}$. The line bundle $A \cong \mathscr{O}_{\mathbf{P}^{1}}(4)$ on $C$ is of degree $\alpha=4$. Consider an extension

$$
0 \rightarrow \mathscr{O}_{C} \rightarrow \mathscr{E} \rightarrow A \rightarrow 0
$$

corresponding to a general $\xi \in \operatorname{Ext}^{1}\left(A, \mathscr{O}_{C}\right)$. It follows that

$$
\mathscr{E} \cong \mathscr{O}_{\mathbf{P}^{1}}(2) \oplus \mathscr{O}_{\mathbf{P}^{1}}(2) \text {. }
$$

Hence, $S=\mathbf{P}(\mathscr{E}) \cong C \times \mathbf{P}^{1} \cong \mathbf{P}^{1} \times \mathbf{P}^{1}$. We identify $C$ with the section of $S$ corresponding to the short exact sequence. It follows that $C \sim C_{0}+2 f$ where $C_{0}$ is a horizontal section of $S$.

7.2. Degeneration. Let $\mathfrak{S}=S \times \Delta$ and $Z=C \times \Delta \subset \mathfrak{S}$. We identify $S$ with the special fiber of $\mathfrak{S} \rightarrow \Delta$. We take $\Gamma=\Gamma_{1}+\cdots+\Gamma_{4}$ on $S$, where each $\Gamma_{i} \sim C_{0}$ is a general horizontal section of $S$. Let $\Gamma_{i} \cap C=\left\{P_{2 i-1}, P_{2 i}\right\}$. Next we specialize the eight relative points $\mathscr{P}_{i} \subset Z$ to $P_{i} \in C$ in a general way.

7.3. Semistability. Let $\widetilde{\mathfrak{S}}$ be the blowup of $\mathfrak{S}$ along the relative points $\mathscr{P}_{i}$. We have to show that, for each $i$, the conormal bundle $\mathscr{N}_{\widetilde{\Gamma}_{i} / \widetilde{\mathfrak{S}}}$ is semistable (of slope $1)$. Denote by $P_{i}^{\prime}$ the image of $\mathscr{P}_{i}$ in the corresponding $\mathbf{P}\left(\mathscr{N}_{\Gamma / \mathfrak{S}}\right) \cong \Gamma \times \mathbf{P}^{1}$. Then, $\mathbf{P}\left(\mathscr{N}_{\widetilde{\Gamma}_{i} / \widetilde{S}}\right)$ is obtained from $\mathbf{P}\left(\mathscr{N}_{\left.\Gamma_{i} / \mathfrak{S}\right)}\right.$ by performing elementary transforms at the points $P_{2 i-1}^{\prime}, P_{2 i}^{\prime}$. If the specialization is general enough, the points $P_{2 i-1}^{\prime}, P_{2 i}^{\prime}$ do

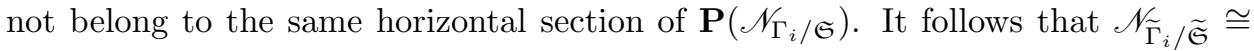
$\mathscr{O}_{\Gamma_{i}}\left(P_{2 i-1}\right) \oplus \mathscr{O}_{\Gamma_{i}}\left(P_{2 i}\right) \cong \mathscr{O}_{\mathbf{P}^{1}}(1) \oplus \mathscr{O}_{\mathbf{P}^{1}}(1)$, which is semistable of slope 1 .

7.4. Invariants. Denote $\mu_{i}=\operatorname{mult}_{\Gamma_{i} / \mathfrak{S}}(\mathscr{C})$. By symmetry, $\mu_{1}=\cdots=\mu_{4}$. Since $\Gamma_{i} \sim C_{0}$, we have the following upper bound:

$$
\mu_{1} \leq \frac{\mu}{4} \leq \frac{d}{4 \cdot 2}
$$

The lower bound from the Basic Lemma is:

$$
\frac{1}{2} \mu_{1} \geq \gamma=3 m-d
$$


Combining $(\sharp)$ and $(b)$, we get:

$$
\frac{1}{2} \cdot \frac{d}{8} \geq 3 m-d \Longleftrightarrow 17 d \geq 48 m .
$$

The bound is sharp:

Example 7.1. The linear system $\mathscr{L}(48,8,17)$ has a unique section, namely the union of 8 curves of degree 6 each passing through 7 points with multiplicity 2 and 1 point with multiplicity 3 ([9], remark on p. 772; [5], Prop. 2.3).

\section{TEN POINTS}

Here we prove the Main Theorem for $n=10$ points.

8.1. Setup. Since $k=3$, we take $C$ to be a smooth cubic in $\mathbf{P}^{2}$. Fix a point $W$ on $C$ such that

$$
9 W \sim 3 H
$$

For example, we can take $W$ to be a Weierstrass point of $C$ (however, later in Section 11 we will require that $3 W \nsim H)$. Let $A=\mathscr{O}_{C}(W)$ which is of degree $\alpha=1$. Since $h^{1}\left(A^{\vee}\right)=1$, there is a unique nontrivial extension

$$
0 \rightarrow \mathscr{O}_{C} \rightarrow \mathscr{E} \rightarrow A \rightarrow 0
$$

The surface $S=\mathbf{P}(\mathscr{E})$ is an indecomposable elliptic ruled surface of degree 1 . The short exact sequence determines a minimal section of $S$ which we identify with $C$. Recall that

$$
-K_{S} \sim 2 C-\pi^{*} A
$$

and

$$
K_{S}^{2}=0
$$

8.2. Degeneration. Let $\mathfrak{S}=S \times \Delta$ and $Z=C \times \Delta \subset \mathfrak{S}$. We identify $S$ with the special fiber of $\mathfrak{S} \rightarrow \Delta$. Next, we take $\Gamma=\Gamma_{1}+\cdots+\Gamma_{5}$, where each $\Gamma_{i}$ is a general section of the pencil $\left|-2 K_{S}\right|$. Each $\Gamma_{i} \equiv 4 C-2 f$ is a smooth elliptic curve and $\Gamma_{i} \rightarrow C$ is the multiplication-by-2 map. Denote $\Gamma_{i} \cap C=\left\{P_{2 i-1}, P_{2 i}\right\}$. Note that $P_{2 i-1}+P_{2 i} \sim 2 A$. Since $3 A \sim H$, we have

$$
\sum P_{i} \sim 10 A \sim A+3 H .
$$

Finally, we specialize the ten relative points $\mathscr{P}_{1}, \ldots, \mathscr{P}_{10}$ in $Z$ to $P_{1}, \ldots, P_{10}$ in a general way such that

$$
\sum \mathscr{P}_{i, t} \sim A+3 H
$$

for any $t \in \Delta$.

8.3. Semistability. We claim that, for each $i, \mathscr{N}_{\widetilde{\Gamma}_{i} / \widetilde{\mathcal{S}}}$ is semistable (of slope 1). Denote by $P_{i}^{\prime}$ the image of $\mathscr{P}_{i}$ in $\mathbf{P}\left(\mathscr{N}_{\Gamma / \mathfrak{S}}\right) \cong \mathbf{P}\left(\mathscr{O}_{\Gamma} \oplus \mathscr{O}_{\Gamma}\right) \cong \Gamma \times \mathbf{P}^{1}$. Now, $\mathbf{P}\left(\mathscr{N}_{\widetilde{\Gamma}_{i} / \widetilde{S}}\right)$ is obtained from $\mathbf{P}\left(\mathscr{N}_{\left.\Gamma_{i} / \mathfrak{S}\right)}\right.$ by performing elementary transforms at the points $P_{2 i-1}^{\prime}, P_{2 i}^{\prime}$. If the specialization is general enough, the points $P_{2 i-1}^{\prime}, P_{2 i}^{\prime}$ do

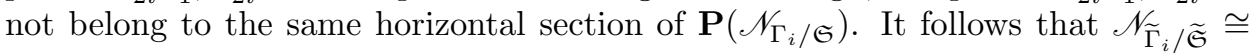
$\mathscr{O}_{\Gamma_{i}}\left(P_{2 i-1}\right) \oplus \mathscr{O}_{\Gamma_{i}}\left(P_{2 i}\right)$, which is semistable of slope 1. 


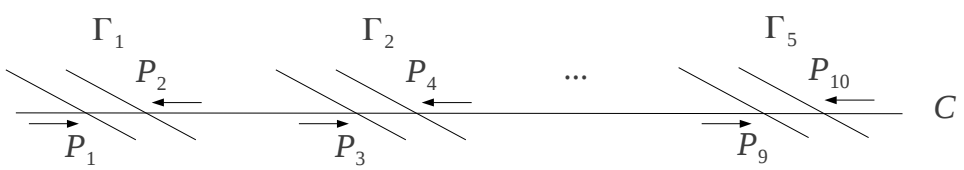

Figure 3. Specialization to $\Gamma(n=10)$

8.4. Invariants. Denote $\mu_{i}=\operatorname{mult}_{\Gamma_{i} / \mathfrak{S}}(\mathscr{C})$. By symmetry, $\mu_{1}=\cdots=\mu_{5}$. Since $\Gamma_{i} \equiv 4 C-2 f$, we have the following upper bound:

$$
\mu_{1} \leq \frac{\mu}{5 \cdot 4} \leq \frac{d}{5 \cdot 4 \cdot 3}
$$

The lower bound from the Basic Lemma is:

$$
\frac{1}{2} \mu_{1} \geq \gamma=19 m-6 d
$$

From $(\sharp)$ and $(b)$, we get:

$$
\frac{1}{2} \cdot \frac{d}{60} \geq 19 m-6 d \Longleftrightarrow 721 d \geq 2280 m
$$

\section{Eleven points}

We prove the Main Theorem for $n=11$ points. This is the first time we will study an interpolation problem $\mathscr{L}_{S}(\mu, \mathfrak{b}, \widehat{m})$ by deforming the underlying surface $S$ itself.

9.1. Setup. As before, $C$ is a smooth cubic in $\mathbf{P}^{2}$. Fix a point $W$ on $C$ such that $9 W \sim 3 H$. Let $A$ be any line bundle of degree $\alpha=2$ on $C$. It is easy to see that for a general $\xi \in \operatorname{Ext}^{1}\left(A, \mathscr{O}_{C}\right)$, the ruled surface $S(C, A, \xi)$ is decomposable of degree 0 . Denote by $C_{(i)}, i=0,1$, the two minimal sections of $S$. It follows that $C \equiv C_{(i)}+f$.

9.2. Degeneration. We construct a relative marked ruled surface $\mathfrak{S}\left(Z, \mathscr{A}, \xi ;\left\{\mathscr{P}_{i j}\right\}\right)$ such that:

- The special fiber $\mathfrak{S}_{0}$ is simply $C \times \mathbf{P}^{1}$.

- The special section $Z_{0}=C_{0} \cup F$; here $C_{0}$ is a horizontal section of $\mathfrak{S}_{0}$ and $F$ is the fiber of $\mathfrak{S}_{0}$ above $W$.

- The relative points $\left\{\mathscr{P}_{i}\right\}$ on $Z$ are such that $\sum \mathscr{P}_{i, t} \sim \mathscr{A}_{t}+k H$ on $Z_{t} \cong C$, for general $t$.

- Each limit point $P_{i}=\mathscr{P}_{i, 0}$ is a general point on $F$.

The construction is done as follows. First, we choose relative points $\overline{\mathscr{P}}_{i}$ in $C \times \Delta$ specializing to $W \times\{0\}$ in a general way. Let

$$
\begin{aligned}
\mathscr{A}^{\prime} & =\mathscr{O}_{C \times \Delta}\left(\sum \overline{\mathscr{P}}_{i}-3 H\right) ; \\
\mathscr{A} & =\mathscr{A}^{\prime} \otimes \mathscr{I}_{W \times\{0\}} .
\end{aligned}
$$

Since $9 W \sim 3 H$, it follows that

$$
\begin{aligned}
& \mathscr{A}_{0}^{\prime} \cong \mathscr{O}_{C}(11 W-3 H) \cong \mathscr{O}_{C}(2 W) ; \\
& \mathscr{A}_{0} \cong \mathscr{O}_{C}(W) \oplus \mathscr{O}_{W}(W) .
\end{aligned}
$$


Consider the following short exact sequence on $C \times\{0\}$ :

$$
0 \rightarrow \mathscr{O}_{C} \rightarrow \mathscr{O}_{C}(W) \oplus \mathscr{O}_{C}(W) \rightarrow \mathscr{O}_{C}(W) \oplus \mathscr{O}_{W}(W) \rightarrow 0
$$

By Proposition 5.4, the sequence can be extended to

$$
0 \rightarrow \mathscr{O}_{C \times \Delta} \rightarrow \mathscr{E} \rightarrow \mathscr{A} \rightarrow 0
$$

with $\mathscr{E}$ locally free. Let $\mathfrak{S}=\mathbf{P}(\mathscr{E})$ and $Z=\mathbf{P}(\mathscr{A})$. Hence $\mathfrak{S}_{0}=\mathbf{P}\left(\mathscr{O}_{C}(W) \oplus\right.$ $\left.\mathscr{O}_{C}(W)\right)=C \times \mathbf{P}^{1}$. Now, $Z \rightarrow C \times \Delta$ is just the blowup of $W \times\{0\}$ with exceptional divisor $F$. Finally, we take $\mathscr{P}_{i}$ to be the strict transform of $\overline{\mathscr{P}}_{i}$ in $Z$.

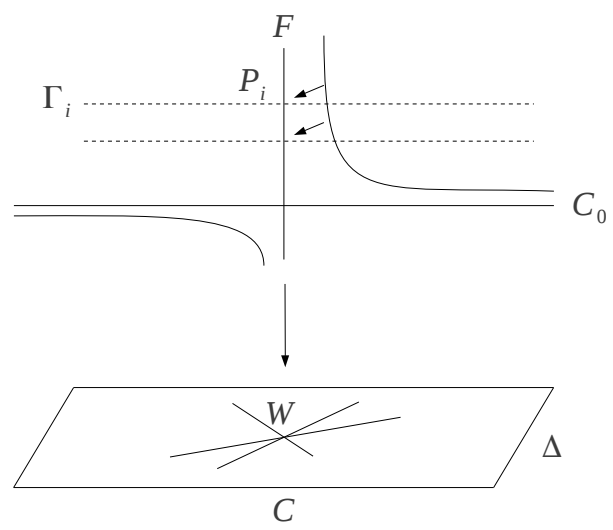

Figure 4. The blowup $Z \rightarrow C \times \Delta$

9.3. Semistability. We take $\Gamma=\Gamma_{1}+\cdots+\Gamma_{11}$ where $\Gamma_{i}$ is the horizontal section of $\mathfrak{S}_{0}=C \times \mathbf{P}^{1}$ through $P_{i}$ (see Figure 4). Consider the blowup $\widetilde{\mathfrak{S}} \rightarrow \mathfrak{S}$ at the relative points $\mathscr{P}_{i}$. We claim that for each $i=1, \ldots, 11, \mathscr{N}_{\widetilde{\Gamma}_{i} / \widetilde{\mathfrak{S}}}$ is indecomposable of degree 1 (hence semistable of slope $1 / 2$ ). First, we will show that $\mathscr{N}_{\Gamma_{i} / \mathfrak{S}}$ is indecomposable of degree 0.

We will need some deformation theory. Let $D=\mathbb{C}[t] / t^{2}$ be the ring of dual numbers. Let $\mathfrak{S}^{\prime}=\mathfrak{S} \times_{\Delta} D$ be viewed as an infinitesimal deformation of $\mathfrak{S}_{0} \cong$ $C \times \mathbf{P}^{1}$ over $D$. We will say that a section $T$ of $\mathfrak{S}_{0}$ is (infinitesimally) unobstructed if and only if $T$ can be extended to a subscheme $T^{\prime}$ of $\mathfrak{S}^{\prime}$ flat over $D$.

Lemma 9.1. Assume the above setting.

(a) $T$ is unobstructed if and only if the following short exact sequence splits:

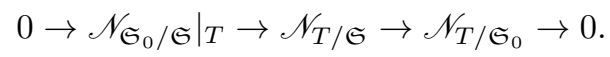

(b) Suppose there are 3 disjoint horizontal sections $T_{1}, T_{2}, T_{3}$ of $\mathfrak{S}_{0} \cong C \times \mathbf{P}^{1}$ that are unobstructed. Then, $\mathfrak{S}^{\prime}$ is an infinitesimally trivial deformation, i.e. $\mathfrak{S}^{\prime} \cong \mathfrak{S}_{0} \times D$. In particular, any section of $\mathfrak{S}_{0}$ is unobstructed.

(c) $C_{0}$ is obstructed.

Proof. (a) This is clear.

(b) We have $\mathfrak{S}^{\prime}=\mathbf{P}\left(\mathscr{E}^{\prime}\right)$ where $\mathscr{E}^{\prime}=\mathscr{E} \otimes D$. For any $i=1,2,3$, the embedding $f_{i}: T_{i}^{\prime} \rightarrow \mathfrak{S}^{\prime}$ induces a surjective morphism $\mathscr{E}^{\prime} \rightarrow \mathscr{L}_{i}^{\prime}$ where $\mathscr{L}_{i}^{\prime}=f_{i}^{*} \mathscr{O}_{\mathfrak{S}^{\prime}}(1)$ is a line bundle on $C^{\prime}=C \times D$. By Nakayama's lemma, for any $i \neq j$, the induced 
map $\mathscr{E}^{\prime} \rightarrow \mathscr{L}_{i}^{\prime} \oplus \mathscr{L}_{j}^{\prime}$ is an isomorphism. Hence $\mathscr{L}_{1}^{\prime} \cong \mathscr{L}_{2}^{\prime} \cong \mathscr{L}_{3}^{\prime} \cong \operatorname{coker}\left(\mathscr{E}^{\prime} \rightarrow\right.$ $\left.\mathscr{L}_{1}^{\prime} \oplus \mathscr{L}_{2}^{\prime} \oplus \mathscr{L}_{3}^{\prime}\right)$, i.e. $\mathfrak{S}^{\prime} \cong \mathfrak{S}_{0} \times D$.

(c) Consider the short exact sequence

$$
\left.0 \rightarrow \mathscr{N}_{Z / \mathfrak{S}}\right|_{C_{0}} \rightarrow \mathscr{N}_{C_{0} / \mathfrak{S}} \rightarrow \mathscr{N}_{C_{0} / Z} \rightarrow 0
$$

where $\left.\mathscr{N}_{Z / \mathfrak{S}}\right|_{C_{0}} \cong \mathscr{O}_{C}(-W)$ and $\mathscr{N}_{C_{0} / Z} \cong \mathscr{O}_{C}(W)$. Since $h^{0}\left(C, \mathscr{O}_{C}(W)\right)=1$, it is clear that $\mathscr{N}_{C_{0} / \mathfrak{S}} ¥ \mathscr{O}_{C} \oplus \mathscr{O}_{C}$. Now consider the short exact sequence from part (a):

$$
\left.0 \rightarrow \mathscr{N}_{\mathfrak{S}_{0} / \mathfrak{S}}\right|_{C_{0}} \rightarrow \mathscr{N}_{C_{0} / \mathfrak{S}} \rightarrow \mathscr{N}_{C_{0} / \mathfrak{S}_{0}} \rightarrow 0
$$

where $\left.\mathscr{N}_{\mathfrak{S}_{0} / \mathfrak{S}}\right|_{C_{0}} \cong \mathscr{O}_{C}$ and $\mathscr{N}_{C_{0} / \mathfrak{S}_{0}} \cong \mathscr{O}_{C}$. It follows that $\mathscr{N}_{C_{0} / \mathfrak{S}_{0}}$ is indecomposable of degree 0 . Hence, $C_{0}$ is obstructed.

Part (c) of the lemma implies that $\mathfrak{S}^{\prime}$ is not an infinitesimally trivial deformation. By part (b) and by symmetry, $\Gamma_{i}$ is obstructed for any $i$. It follows that the conormal bundle $\mathscr{N}_{\Gamma_{i} / \mathfrak{S}}$ is indecomposable of degree 0 .

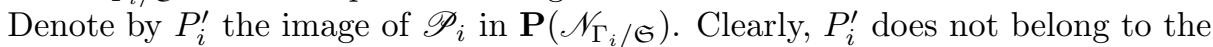
unique minimal section of $\mathbf{P}\left(\mathscr{N}_{\left.\Gamma_{i} / \mathfrak{S}\right)}\right)$ (because $\mathscr{P}_{i}$ meets $\mathfrak{S}_{0}$ transversely). Finally, $\mathbf{P}\left(\mathscr{N}_{\widetilde{\Gamma}_{i} / \widetilde{S}}\right)$ is obtained from $\mathbf{P}\left(\mathscr{N}_{\left.\Gamma_{i} / \mathfrak{S}\right)}\right.$ by performing an elementary transform at $P_{i}^{\prime}$. It follows that $\mathscr{N}_{\widetilde{\Gamma}_{i} / \widetilde{\mathfrak{S}}}$ is indecomposable of degree 1 .

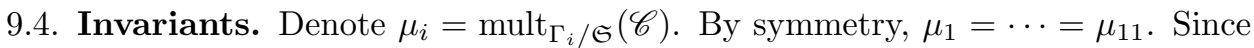
$\Gamma_{i} \equiv C_{0}$, we have the following upper bound:

$$
\mu_{1} \leq \frac{\mu}{11} \leq \frac{d}{11 \cdot 3}
$$

The lower bound from the Basic Lemma is:

$$
\frac{1}{2} \mu_{1} \geq \gamma=10 m-3 d
$$

Combining $(\sharp)$ and $(b)$, we get:

$$
\frac{1}{2} \cdot \frac{d}{33} \geq 10 m-3 d \Longleftrightarrow 199 d \geq 660 m \text {. }
$$

Remark 9.2. It might also be profitable to study the behavior of $\mathscr{C}$ along the fiber $F$. We have $\mathscr{N}_{\widetilde{F} / \widetilde{S}} \cong \mathscr{O}_{\mathbf{P}^{1}}(10) \oplus \mathscr{O}_{\mathbf{P}^{1}}(1)$, which follows from the split exact sequence

$$
\left.0 \rightarrow \mathscr{N}_{\widetilde{Z} / \widetilde{\mathfrak{S}}}\right|_{\widetilde{F}} \rightarrow \mathscr{N}_{\widetilde{F} / \widetilde{\mathfrak{S}}} \rightarrow \mathscr{N}_{\widetilde{F} / \widetilde{Z}} \rightarrow 0
$$

with $\left.\mathscr{N}_{\widetilde{Z} / \widetilde{\mathfrak{S}}}\right|_{\widetilde{F}} \cong \mathscr{O}_{\mathbf{P}^{1}}(10)$ and $\mathscr{N}_{\widetilde{F} / \widetilde{Z}} \cong \mathscr{N}_{F / Z} \cong \mathscr{O}_{\mathbf{P}^{1}}(1)$. The fact that $\mathscr{N}_{\widetilde{F} / \widetilde{\mathfrak{S}}}$ is unstable causes certain multiplicity and tangency conditions on the limit curve $\mathscr{C}_{0}$ at the point $W=C_{0} \cap F$. A more careful analysis of the situation is beyond the scope of this paper.

\section{TWELVE POINTS}

In this section we prove the Main Theorem for $n=12$. This case is similar to $n=10$.

10.1. Setup. As before, $C$ is a smooth cubic in $\mathbf{P}^{2}$. Fix a point $W$ on $C$ such that $9 W \sim 3 H$. Take $A=\mathscr{O}_{C}(3 W)$ which is of degree $\alpha=3$. It is easy to see that for a general $\xi \in \operatorname{Ext}^{1}\left(A, \mathscr{O}_{C}\right), S(C, A, \xi)$ is an indecomposable elliptic ruled surface of degree 1. It follows that $C \equiv C_{0}+f$ where $C_{0}$ is a minimal section of $S$. 
10.2. Degeneration. Let $\mathfrak{S}=S \times \Delta$ and $Z=C \times \Delta \subset \mathfrak{S}$. We identify $S$ with the special fiber of $\mathfrak{S}$. Take $\Gamma=\Gamma_{1}+\Gamma_{2}$ where each $\Gamma_{i}$ is a general section of the pencil $\left|-2 K_{S}\right|$. In particular, $\Gamma_{i} \cdot C=\left(4 C_{0}-2 f\right) \cdot\left(C_{0}+f\right)=6$. Let $C \cap \Gamma_{1}=\left\{P_{1}, \ldots, P_{6}\right\}$ and $C \cap \Gamma_{2}=\left\{P_{7}, \ldots, P_{12}\right\}$. It follows that

$$
\sum P_{i} \sim 4 A \sim A+3 H
$$

Next, we specialize $\mathscr{P}_{i}$ in $Z$ to $P_{i}$ in a general way such that

$$
\sum \mathscr{P}_{i, t} \sim A+3 H
$$

for any $t \in \Delta$.

10.3. Semistability. We have to show that if the specialization of the points $\mathscr{P}_{i}$ is general enough, the conormal bundle $\mathscr{N}_{\widetilde{\Gamma}_{i} / \widetilde{\mathfrak{S}}}$ is semistable (of slope 3). Since semistability is an open property, it suffices to describe a particular specialization for which $\mathscr{N}_{\widetilde{\Gamma}_{i} / \widetilde{\mathfrak{S}}}$ is semistable. In fact, we claim that we can specialize the points in such a way that

$$
\mathscr{N}_{\widetilde{\Gamma}_{1} / \widetilde{\mathfrak{S}}} \cong \mathscr{O}_{\Gamma_{1}}\left(P_{1}+P_{2}+P_{3}\right) \oplus \mathscr{O}_{\Gamma_{1}}\left(P_{4}+P_{5}+P_{6}\right)
$$

and similarly for $\Gamma_{2}$. This can be achieved by moving the triples of points $\left\{\mathscr{P}_{3 i-2}, \mathscr{P}_{3 i-1}, \mathscr{P}_{3 i}\right\}$ "in parallel" while being assigned to the same section of the pencil $\left|-2 K_{S}\right|$.

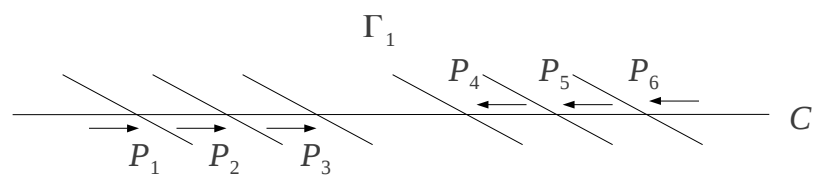

FiguRE 5. Specialization to $\Gamma(n=12)$

10.4. Invariants. Denote $\mu_{i}=\operatorname{mult}_{\Gamma_{i} / \mathfrak{S}}(\mathscr{C})$. By symmetry, $\mu_{1}=\mu_{2}$. Since $\Gamma_{i} \equiv$ $4 C_{0}-2 f$, we have the following upper bound:

$$
\mu_{1} \leq \frac{\mu}{2 \cdot 4} \leq \frac{d}{2 \cdot 4 \cdot 3}
$$

The lower bound from the Basic Lemma is:

$$
\frac{1}{2} \mu_{1} \geq \gamma=7 m-2 d .
$$

Combining $(\sharp)$ and $(b)$, we get:

$$
\frac{1}{2} \cdot \frac{d}{24} \geq 7 m-2 d \Longleftrightarrow 97 d \geq 336 m .
$$




\section{A REFinement}

Here we prove a certain refinement of the Main Theorem in the case of $n=10,11$ and 12 points. We work in the setting of the previous sections. The idea is to show that, under some additional assumptions, the inequality $(b)$ can be replaced by a stronger inequality (bb). First, we have:

Lemma 11.1. Let $n=10,11$ or 12 . Consider the degeneration described above for the particular value of $n$. Let $\mathfrak{b}:=\mathscr{B}_{0}=\mathscr{O}_{C}\left(\sum m P_{i}-d H\right)$.

(a) We have $\mathfrak{b}-b W \sim d(3 W-H)$. In particular, $3 \mathfrak{b} \sim 3 b W$.

(b) Assume there is an equality in (b), i.e. $\frac{1}{2} \mu_{1}=\gamma$. Then $\mathfrak{b} \sim b W$.

Proof. (a) We have $\sum P_{i} \sim n W$. Therefore

$$
\begin{aligned}
\mathfrak{b}-b W & \sim(n m W-d H)-(n m-3 d) W \\
& \sim d(3 W-H) .
\end{aligned}
$$

Since $9 W \sim 3 H$, it follows that $3 \mathfrak{b} \sim 3 b W$.

(b) Assume $\frac{1}{2} \mu_{1}=\gamma$. By the Basic Lemma, there is an injective morphism:

$$
\mathscr{O}_{\widetilde{\Gamma}_{1}}(-\widetilde{\mathscr{C}}) \hookrightarrow \operatorname{Sym}^{2 \gamma} \mathscr{N}_{\widetilde{\Gamma}_{1} / \widetilde{S}}
$$

Now the idea is to show that the vector bundle on the right hand side decomposes as a direct sum of line bundles of the same degree as $\mathscr{O}_{\widetilde{\Gamma}_{1}}(-\widetilde{\mathscr{C}})$. It will follow that $\mathscr{O}_{\widetilde{\Gamma}_{1}}(-\widetilde{\mathscr{C}})$ is isomorphic to one of the summands. Below we consider each case for $n$ separately.

Case $n=10$. Here $\mathscr{O}_{\widetilde{\Gamma}_{1}}(-\widetilde{\mathscr{C}})$ is of degree $2 \gamma$. More precisely, since $\mathscr{O}_{\Gamma_{1}}(C) \cong$ $\mathscr{O}_{\Gamma_{1}}\left(P_{1}+P_{2}\right)$, we have:

$$
\mathscr{O}_{\widetilde{\Gamma}_{1}}(-\widetilde{\mathscr{C}}) \cong \mathscr{O}_{\Gamma_{1}}\left(-m\left(P_{1}+P_{2}\right)+\mathfrak{b} f\right) .
$$

Recall that $\mathscr{N}_{\widetilde{\Gamma}_{1} / \widetilde{\mathfrak{S}}} \cong \mathscr{O}_{\Gamma_{1}}\left(P_{1}\right) \oplus \mathscr{O}_{\Gamma_{1}}\left(P_{2}\right)$. Therefore:

$$
\operatorname{Sym}^{2 \gamma} \mathscr{N}_{\widetilde{\Gamma}_{1} / \widetilde{\mathfrak{S}}} \cong \bigoplus_{i+j=2 \gamma} \mathscr{O}_{\Gamma_{1}}\left(i P_{1}+j P_{2}\right) \text {. }
$$

It follows that $\mathscr{O}_{\widetilde{\Gamma}_{1}}(-\widetilde{\mathscr{C}})$ must be isomorphic to one of the summands. Hence $\mathfrak{b} f \sim i P_{1}+j P_{2}$ on $\Gamma_{1}$ for some $i, j$ with $i+j=4 b$. Since $\Gamma_{1} \rightarrow C$ is an isogeny of degree 4 , it follows that $4 \mathfrak{b} \sim i P_{1}+j P_{2}$ on $C$. By symmetry, $4 \mathfrak{b} \sim j P_{1}+i P_{2}$. Therefore, $8 \mathfrak{b} \sim 4 b\left(P_{1}+P_{2}\right) \sim 8 b W$ on $C$. Since $3 \mathfrak{b} \sim 3 b W$ and $\operatorname{gcd}(8,3)=1$, it follows that $\mathfrak{b} \sim b W$.

Case $n=11$. Here $\mathscr{O}_{\widetilde{\Gamma}_{1}}(-\widetilde{\mathscr{C}})$ is of degree $\gamma$. More precisely, since $\mathscr{O}_{\Gamma_{1}}\left(Z_{0}\right) \cong$ $\mathscr{O}_{\Gamma_{1}}\left(P_{1}\right)$, we find:

$$
\mathscr{O}_{\widetilde{\Gamma}_{1}}(-\widetilde{\mathscr{C}}) \cong \mathscr{O}_{\Gamma_{1}}\left(-m P_{1}+\mathfrak{b} f\right) \cong \mathscr{O}_{C}(-m W+\mathfrak{b})
$$

Recall that $\mathscr{N}_{\widetilde{\Gamma}_{1} / \widetilde{S}}$ is indecomposable of degree 1 (determinant $W$ ). Using results in [1], one can show that $\operatorname{Sym}^{2 \gamma}\left(\mathscr{N}_{\widetilde{\Gamma}_{1} / \widetilde{\mathfrak{S}}}\right)$ is a direct sum of line bundles of the form $\mathscr{O}_{C}\left(\gamma W+L_{i}\right)$ where $L_{i}$ are line bundles with $L_{i}^{\otimes 2} \cong \mathscr{O}_{C}$. We conclude that $\mathscr{O}_{\widetilde{\Gamma}_{1}}(-\widetilde{\mathscr{C}})$ is isomorphic to one of the summands. It follows that $2 \mathfrak{b} \sim 2 b W$. Since $3 \mathfrak{b} \sim 3 b W$ and $\operatorname{gcd}(2,3)=1$, we conclude that $\mathfrak{b} \sim b W$. 
Case $n=12$. This is similar to the case of ten points. We leave the details to the reader.

The following result is a refinement of the Main Theorem. Note that it only applies when $3 \nmid d$.

Proposition 11.2. Let $n=10,11$ or 12 . If $\mathscr{L}(d, n, m)$ is nonempty and $3 \nmid d$, then $\kappa_{n} \geq 0$ with

$$
\begin{array}{r}
\kappa_{10}=721 d-2280 m-60 \\
\kappa_{11}=199 d-660 m-33 \\
\kappa_{12}=97 d-336 m-24 .
\end{array}
$$

Proof. Fix $W$ so that $9 W \sim 3 H$ but $3 W \nsim H$. Since $3 \nmid d$, part (a) of the lemma implies that $\mathfrak{b} \nsim b W$. From part (b), we get:

$$
\frac{1}{2} \mu_{1} \geq \gamma+\frac{1}{2}
$$

Finally, $(\sharp)$ together with (bb) imply the desired inequality.

Corollary 11.3. The following linear systems $\mathscr{L}(d, n, m)$ with $v=-1$ are empty, hence nonspecial:

\begin{tabular}{rrr|r|rrrr|r|rr}
$d$ & $n$ & $m$ & $\chi_{\mathbf{P}^{2}}$ & $\mu$ & $\epsilon$ & $b$ & $\widehat{m}$ & $\chi_{S}$ & $\gamma$ & $\kappa_{n}$ \\
\hline 1499 & 10 & 474 & 0 & 499 & 2 & 243 & 25 & 0 & 12 & -1 \\
778 & 10 & 246 & 0 & 259 & 1 & 126 & 13 & 0 & 6 & -2 \\
428 & 11 & 129 & 0 & 142 & 2 & 135 & 13 & 0 & 6 & -1 \\
229 & 11 & 69 & 0 & 76 & 1 & 72 & 7 & 0 & 3 & -2 \\
215 & 12 & 62 & 0 & 71 & 2 & 99 & 9 & 0 & 4 & -1 \\
118 & 12 & 34 & 0 & 39 & 1 & 54 & 5 & 0 & 2 & -2
\end{tabular}

Remark 11.4. The assumption $3 \nmid d$ in the proposition cannot be dropped. For example, consider the nonempty linear system $\mathscr{L}(57,10,18)$ (with $v=0, \kappa_{10}=-3$ and $3 \mid d)$. Unfortunately, it is not clear to us how to extend the proposition in the case $3 \mid d$. Our discussion will not be complete without mentioning the following interesting open problems: $\mathscr{L}(2220,10,702), \mathscr{L}(627,11,189)$ and $\mathscr{L}(312,12,90)$ (with $v=0, \kappa_{n}=0$ and $3 \mid d$ ).

\section{The REMAINing CASE}

Here we prove the Main Theorem in the case when $k \geq 3, \alpha$ is even, $\alpha \mid 2 k$. This generalizes the case of eleven points (in fact, the proof can also be applied in the case of eight points).

12.1. Setup. Let $C$ be a smooth plane curve of degree $k$. We will make the following assumption: there is a divisor $W=W_{1}+\cdots+W_{\frac{\alpha}{2}}$ on $C$, where $W_{i}$ 's are distinct points, such that

$$
\frac{2 k^{2}}{\alpha} W \sim k H \text {. }
$$

Here is one way to construct such a curve. Fix a line $\ell \subset \mathbf{P}^{2}$ and let $W_{1}, \ldots, W_{\frac{\alpha}{2}}$ be distinct points on $\ell$. Now, take $C$ to be any smooth curve of degree $k$ which is tangent to $\ell$ to order $\frac{2 k}{\alpha}$ at each of the points $W_{i}$. This is possible since $\alpha \mid 2 k$. It follows that $\frac{2 k}{\alpha} W \sim H$, which satisfies the assumption. 
12.2. Degeneration. It will be convenient to re-index the $n$ relative points as $\left\{\mathscr{P}_{i j}\right\}$ where $i=1, \ldots, \frac{2 n}{\alpha}$ and $j=1, \ldots, \frac{\alpha}{2}$. We will construct a relative marked ruled surface $\mathfrak{S}\left(Z, \mathscr{A}, \xi ;\left\{\mathscr{P}_{i j}\right\}\right)$ such that:

- The special fiber $\mathfrak{S}_{0}$ is simply $C \times \mathbf{P}^{1}$.

- The special section $Z_{0}=C_{0} \cup F_{1} \cup \cdots \cup F_{\frac{\alpha}{2}}$; here $C_{0}$ is a horizontal section of $\mathfrak{S}_{0}$ and $F_{j}$ is the fiber of $\mathfrak{S}_{0}$ above $W_{j}$, for each $j$.

- The relative points $\left\{\mathscr{P}_{i j}\right\}$ on $Z$ are such that $\sum \mathscr{P}_{i j, t} \sim \mathscr{A}_{t}+k H$ on $Z_{t} \cong C$, for general $t$.

- Each limit point $P_{i j}=\mathscr{P}_{i j, 0}$ is a general point on the fiber $F_{j}$.

The construction generalizes the case of eleven points. Namely, we first choose relative points $\overline{\mathscr{P}}_{i j}$ in $C \times \Delta$ specializing to $W_{j} \times\{0\}$ in a general way. Next, let $\mathscr{A}^{\prime}=\mathscr{O}_{C \times \Delta}\left(\sum \mathscr{P}_{i j}-k H\right)$ and $\mathscr{A}=\mathscr{A}^{\prime} \otimes I_{W \times\{0\}}$. It follows that

$$
\begin{aligned}
& \mathscr{A}_{0}^{\prime} \cong \mathscr{O}_{C}\left(\frac{2 n}{\alpha} W-k H\right) \cong \mathscr{O}_{C}(2 W) ; \\
& \mathscr{A}_{0} \cong \mathscr{O}_{C}(W) \oplus \mathscr{O}_{W}(W) .
\end{aligned}
$$

Consider the following short exact sequence on $C \times\{0\}$ :

$$
0 \rightarrow \mathscr{O}_{C} \rightarrow \mathscr{O}_{C}(W) \oplus \mathscr{O}_{C}(W) \rightarrow \mathscr{O}_{C}(W) \oplus \mathscr{O}_{W}(W) \rightarrow 0
$$

Note that $h^{0}\left(C, \mathscr{O}_{C}(W)\right)=1$, so the sequence is essentially unique. By Proposition 5.4, the sequence can be extended to

$$
0 \rightarrow \mathscr{O}_{C \times \Delta} \rightarrow \mathscr{E} \rightarrow \mathscr{A} \rightarrow 0
$$

over $C \times \Delta$, where $\mathscr{E}$ is locally free. Finally, we take $\mathfrak{S}=\mathbf{P}(\mathscr{E})$ and $Z=\mathbf{P}(\mathscr{A})$. It follows that $\mathfrak{S}_{0} \cong \mathbf{P}\left(\mathscr{O}_{C} \oplus \mathscr{O}_{C}\right) \cong C \times \mathbf{P}^{1}$. We take $\mathscr{P}_{i j}$ to be the strict transform of the $\overline{\mathscr{P}}_{i j}$ on the blowup $Z \rightarrow C \times \Delta$ at $W \times\{0\}$.

Next, we will distinguish between two cases: $\alpha=2$ and $\alpha \geq 4$.

12.3. Semistability $(\alpha=2)$. Let $\Gamma_{i}$ be the horizontal section of $\mathfrak{S}_{0}$ through $P_{i, 1}$. Just as in the case of eleven points, we can show that the conormal bundle $\mathscr{N}_{\widetilde{\Gamma}_{i} / \widetilde{\mathfrak{S}}}$ is semistable of slope $1 / 2$.

12.4. Second degeneration $(\alpha \geq 4)$. In this case, we perform another degeneration on the trivial ruled surface $S=C \times \mathbf{P}^{1}$. Fix $\frac{2 n}{\alpha}$ general horizontal sections $\Gamma_{1}, \ldots, \Gamma_{\frac{2 n}{\alpha}}$ of $S$. Let $P_{i j}=\Gamma_{i} \cap F_{j}$ for $i=1, \ldots, \frac{2 n}{\alpha}$ and $j=1, \ldots, \frac{\alpha}{2}$. We specialize the $n$ relative points $\mathscr{P}_{i j}$ to $P_{i j}$ by "sliding" them along the corresponding fibers $F_{j}$, in a general way.

12.5. Semistability $(\alpha \geq 4)$. Denote by $\widetilde{S \times \Delta}$ the blowup of $S \times \Delta$ at the relative points $\mathscr{P}_{i j}$ constructed in the previous step. Now, $\mathbf{P}\left(\mathscr{N}_{\widetilde{\Gamma}_{i} / \widetilde{S \times \Delta}}\right)$ is obtained from $\mathbf{P}\left(\mathscr{N}_{\Gamma_{i} / S \times \Delta}\right)=\mathbf{P}\left(\mathscr{O}_{C} \oplus \mathscr{O}_{C}\right)$ by applying $\alpha / 2$ elementary transforms at general points on the fixed fibers through $W_{1}, \ldots, W_{\frac{\alpha}{2}}$. Since $\alpha / 2 \geq 2$, it follows that the resulting vector bundle is semistable of slope $\alpha / 4$.

12.6. Invariants. The computation of invariants was carried out in Section 6 . 


\section{AN AUXILIARY LEMMA}

The lemma below was used in the proof of the Main Theorem. Let $n=k^{2}+\alpha$ where $k>0$ and $\alpha>0$. Consider the matrix

$$
M_{1}=\left[\begin{array}{cc}
k & n \\
1 & k
\end{array}\right] .
$$

For any positive integer $i$, define

$$
M_{i}=\left[\begin{array}{cc}
p_{i} & q_{i} \\
r_{i} & p_{i}
\end{array}\right]=\alpha^{-\lfloor i / 2\rfloor}\left(M_{1}\right)^{i} .
$$

Note that $\operatorname{det}\left(M_{2 i-1}\right)=-\alpha$ and $\operatorname{det}\left(M_{2 i}\right)=1$.

For example,

$$
p_{1}=k ; \quad q_{1}=n ; \quad r_{1}=1 ;
$$

and

$$
p_{2}=\frac{n+k^{2}}{\alpha} ; \quad q_{2}=\frac{2 n k}{\alpha} ; \quad r_{2}=\frac{2 k}{\alpha} .
$$

The ratios $p_{i} / r_{i}$ and $q_{i} / p_{i}$ have natural expansions as continued fractions approximating $\sqrt{n}$. In particular,

$$
\frac{q_{2}}{p_{2}}=c_{n}^{(1)} \quad \text { and } \quad \frac{q_{4}}{p_{4}}=c_{n}^{(2)}
$$

are precisely the constants in Theorem 3.1 and the Main Theorem.

Lemma 13.1. Let $d$ and $m$ be any real numbers.

(a) The following are equivalent:

$$
\frac{\alpha}{2} \frac{d}{p_{1}} \geq q_{1} m-p_{1} d \quad \Longleftrightarrow \quad p_{2} d-q_{2} m \geq 0 .
$$

(b) The following are equivalent:

$$
\frac{1}{2} \frac{d}{q_{2}} \geq p_{2} m-r_{2} d \quad \Longleftrightarrow \quad p_{4} d-q_{4} m \geq 0
$$

Proof. (a) Multiply both sides by $2 p_{1}$ and substitute $\alpha=-p_{1}^{2}+q_{1} r_{1}$ :

$$
\left(\alpha+2 p_{1}^{2}\right) d \geq 2 p_{1} q_{1} m \Longrightarrow \underbrace{\left(p_{1}^{2}+q_{1} r_{1}\right)}_{\alpha p_{2}} d \geq \underbrace{2 p_{1} q_{1}}_{\alpha q_{2}} m .
$$

(b) Multiply both sides by $2 q_{2}$ and substitute $1=p_{2}^{2}-q_{2} r_{2}$ :

$$
\left(1+2 q_{2} r_{2}\right) d \geq 2 p_{2} q_{2} m \Longrightarrow \underbrace{\left(p_{2}^{2}+q_{2} r_{2}\right)}_{p_{4}} d \geq \underbrace{2 p_{2} q_{2}}_{q_{4}} m .
$$

\section{ACKNowledgments}

The author is grateful to E. Cotterill and the anonymous referee for valuable suggestions for improving the paper. 


\section{REFERENCES}

[1] M. F. Atiyah, Vector bundles over an elliptic curve, Proc. London Math. Soc. (3) 7 (1957), 414-452. MR0131423(24 \#A1274)

[2] Paul Biran, Constructing new ample divisors out of old ones, Duke Math. J. 98 (1999), no. 1, 113-135, DOI 10.1215/S0012-7094-99-09803-4. MR.1687571(2000d:14047)

[3] Ciro Ciliberto, Olivia Dumitrescu, Rick Miranda, and Joaquim Roé, Emptiness of homogeneous linear systems with ten general base points, Classification of algebraic varieties, EMS Ser. Congr. Rep., Eur. Math. Soc., Zürich, 2011, pp. 189-195, DOI 10.4171/007-1/8. MR2779472 (2012f:14008)

[4] Ciro Ciliberto and Rick Miranda, Homogeneous interpolation on ten points, J. Algebraic Geom. 20 (2011), no. 4, 685-726, DOI 10.1090/S1056-3911-2011-00545-6. MR.2819673 (2012h:14013)

[5] Ciro Ciliberto and Rick Miranda, Linear systems of plane curves with base points of equal multiplicity, Trans. Amer. Math. Soc. 352 (2000), no. 9, 4037-4050, DOI 10.1090/S00029947-00-02416-8. MR:1637062(2000m:14006)

[6] Thomas Eckl, Ciliberto-Miranda degenerations of $\mathbb{C P}^{2}$ blown up in 10 points, J. Pure Appl. Algebra 215 (2011), no. 4, 672-696, DOI 10.1016/j.jpaa.2010.06.016. MR2738381 (2011m:14063)

[7] Robert Friedman, Algebraic surfaces and holomorphic vector bundles, Universitext, SpringerVerlag, New York, 1998. MR1600388 (99c:14056)

[8] Robin Hartshorne, Algebraic geometry, Springer-Verlag, New York, 1977. Graduate Texts in Mathematics, No. 52. MR0463157 (57 \#3116)

[9] Masayoshi Nagata, On the 14-th problem of Hilbert, Amer. J. Math. 81 (1959), 766-772. MR0105409 (21 \#4151)

[10] Ivan Petrakiev, Multiple points in $\mathbf{P}^{2}$ and degenerations to elliptic curves, Proc. Amer. Math. Soc. 137 (2009), no. 1, 65-71, DOI 10.1090/S0002-9939-08-09540-3. MR2439426 (2009f:14014)

[11] J. Le Potier, Lectures on vector bundles, Cambridge Studies in Advanced Mathematics, vol. 54, Cambridge University Press, Cambridge, 1997. Translated by A. Maciocia. MR 1428426 (98a:14019)

Jersey City, New Jersey

E-mail address: ivan.petrakiev@gmail.com 